\title{
The Grade of Left Ventricular Diastolic Dysfunction as a Predictive Factor for Permanent Atrial Fibrillation
}

\author{
Cerghizan Anda, Keresztesi Arthur Atilla*, Bataga Simona, Tilea loan
}

University of Medicine and Pharmacy of Tirgu Mures, Romania

The objective of this study was to find a link between the grade of left ventricular diastolic dysfunction (LVDD) and the progression to permanent non-valvular atrial fibrillation (AF), in a group of patients with left ventricular diastolic dysfunction and paroxysmal or persistent atrial fibrillation. Methods: A bidirectional study on 57 patients meeting the inclusion criteria was conducted; each patient was admitted in a university -based hospital between January 1st - June, 30, 2013, with a follow up 3 and 6 months later. Permanent atrial fibrillation development was followed. Results: Out of the 57 patients, 23 had paroxysmal AF and 34 were with persistent AF. After six months, 21 patients progressed to permanent $\mathrm{AF}$, representing $36.84 \%$ of the total patients. Female patients with age over 65 had more often atrial fibrillation, but more men progressed to a sustained form of AF. No statistically significant difference regarding the grade of diastolic dysfunction, the left atrial size or volume, or the left ventricular ejection fraction was observed between the patients with progression, compared to those without progression. Conclusions: The grade of left ventricular diastolic dysfunction did not prove to be a predictive factor for permanent atrial fibrillation, neither the left atrial size or volume, or the left ventricular ejection fraction.

Keywords: left ventricular diastolic dysfunction, left atrial volume, atrial fibrillation

Received: 9 December 2014 / Accepted: 25 April 2015

\section{Introduction}

Atrial fibrillation (AF) is a cardiac arrhythmia with a continuous increasing prevalence in the last decades and in the future $20-30$ years, due the ageing of the general population. It is estimated that in the US alone the number of people suffering of AF will increase from 1.2 million people today to 2.6 million cases in 2030 [1].

The majority of people suffering from this arrhythmia are over 75 years of age [2], and have frequently associated others comorbid states like arterial hypertension $(83 \%)$, ischemic heart disease (64\%), hyperlipidemia (62\%), heart failure (51\%).

For clinical management of patients with AF we used the ESC Guidelines for the management of atrial fibrillation as follows [3]:

1. „Every patient who presents with AF for the first time was considered a patient with first diagnosed AF, irrespective of the duration of the arrhythmia or the presence and severity of AF-related symptoms".

2. „Paroxysmal AF is a self-terminating rhythm disturbance, usually within $48 \mathrm{~h}$ ".

3. „Persistent $\mathrm{AF}$ - an $\mathrm{AF}$ episode either lasts longer than 7 days or requires termination by cardioversion, either with drugs or by direct current cardioversion (DCC)".

4. „Long-standing persistent AF has lasted for $\geq 1$ year when it is decided to adopt a rhythm control strategy".

5. „Permanent AF is said to exist when the presence of the arrhythmia is accepted by the patient (and physician)".
6. „Silent AF (asymptomatic) may manifest as an AFrelated complication (ischaemic stroke or tachycardiomyopathy) or may be diagnosed by an opportunistic ECG”.

Patients with heart failure are more often predisposed to develop atrial fibrillation, and this risk increases even more at patients with preserved ejection fraction. Left ventricular diastolic dysfunction (LVDD) has many common risks factors with $\mathrm{AF}$ and has a proarrhythmic role due to atrial remodeling $[4,5]$.

The aim of this study was to find possible associations between the grade of left ventricular diastolic dysfunction, the size of the left atria and the progression to permanent non-valvular atrial fibrillation, in a group of patients with LVDD and paroxysmal or persistent atrial fibrillation.

\section{Methods}

Between 2013 January $1^{\text {st }}$ and June $30^{\text {th }}$ we conducted a bidirectional observational study using a cohort of 889 consecutive patients with cardiovascular diseases, admitted in a university based-hospital; 57 patients $(28$ men and 29 women) were selected and called in for follow-up, from the total of 143 patients diagnosed with diastolic dysfunction.

The protocol study was reviewed and approved by the Ethics Committee of the University of Medicine and Pharmacy of Tirgu Mures and has been performed in the accordance with standards of the Declaration of Helsinki/ local regulations. All subjects provided written informed consent. Study was performed on a background of guideline-based medical care. 


\section{Selection criteria}

- Paroxysmal or persistent atrial fibrillation diagnosed via rest electrocardiography (ECG) or 24 hour Holter ECG;

- Echocardiographic examination during the first admission, patients in sinus rhythm as follows:

- Left atrium (LA) area and volume;

- LA volume/body surface area (BSA);

- left ventricular (LV) ejection fraction - method used: modified Simpson rule (LV endocard marking in apical 2 and 4 chamber);

- Grade of diastolic dysfunction;

- Absence of valvular heart disease.

A number of 64 patients met the inclusion criteria and were called in for a follow-up at 3, respectively 6 months. Fifty seven of them agreed to participate in the current study.

During each follow-up exam, all studied patients underwent a rest and Holter ECG examination. At 3 months all the patients underwent an echocardiographic evaluation, with the specification that all of them were in sinus rhythm. After the second follow-up admission, permanent atrial fibrillation was diagnosed based on the Holter ECG evaluations and the patient refusal for an electrical or drug conversion to sinus rhythm.

Grades of left ventricular diastolic dysfunction were defined as:

- grade 1 when the ratio of peak early filling (E wave) and late diastolic filling (A wave) is $<1$;

- grade 2 - E/A ratio is between $1-2, \mathrm{Em} / \mathrm{Am}<1$ and $\mathrm{E} / \mathrm{Em}>15$, where Em represents the diastolic relaxation and Am represents atrial contraction via tissue Doppler echocardiography.

- grade 3 as E/A ratio $>2$.

Data collected were included in an electronical database using Microsoft Excel 2010 software. Statistical analysis was performed using Epi Info software; Fisher's exact test, Chi square Yates corrected Chi square, $\mathrm{T}$ test and Mann Whitney tests were used, with a confidence level of $95 \%$. A p value $<0.05$ was considered statistically significant. Continuous variables are reported as mean \pm standard deviation (SD) and categorical variables as observed number of patients.

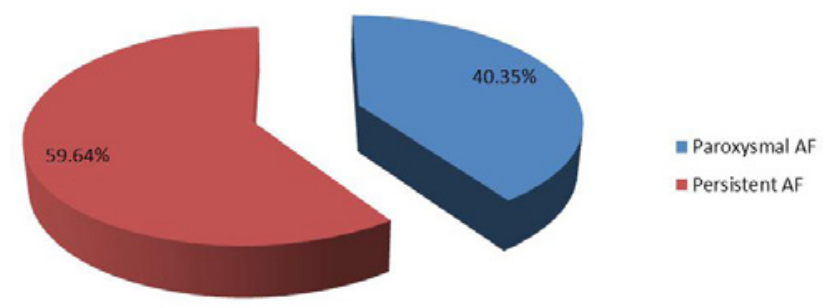

Fig. 1. Distribution of atrial fibrillation according to the type

\section{Results}

The mean age of the 57 participants in the study was $65 \pm$ 11 years (range from 34 to 84 years), 28 patients were male and 29 female. The mean age of the male patients was 61 \pm 12 years, while women had a mean age of $68 \pm 9$ years ( $p$ 0.036), male patients were statistically younger than female patients with LVDD.

The main symptoms at first admission were fatigue $(89.47 \%)$, dyspnea $(73.68 \%)$, irregular heart beat $(31.57 \%)$, chest discomfort $(22.8 \%)$ and syncope (12.67\%).

As associated diseases, arterial hypertension was the most frequent (92.98\%), followed by hypercholesterolemia $(33.33 \%)$, diabetes mellitus $(29.82 \%)$, coronary artery disease (28.07\%) and hypertriglyceridemia (26.31\%).

Out of the 57 patients, 23 had paroxysmal AF and 34 were with persistent $\mathrm{AF}$ (figure 1).

From the patient medical records, left atrium area and volume, left ventricular ejection fraction and the E/A ratio were collected; these measurements were performed while the patients were in sinus rhythm during the first admission (spontaneous or converted sinus rhythm) (table I). These measurements were repeated at the 3 months followup and no statistically significant difference was observed compared to baseline characteristics.

After six months, 21 patients progressed to permanent AF, representing $36.84 \%$ of the total patients. Seven of them had paroxysmal AF at baseline and 14 had persistent $\mathrm{AF}$ at baseline (figure 2).

The 21 patients that progressed to permanent atrial fibrillation had the mean age of $62 \pm 11$ years ( $p 0.16), 12$ of them were male $(57.14 \%, \mathrm{p} 0.51)$, they had a slightly bigger mean left atrial diameter than those without progression $(22.04 \pm 6.72 \mathrm{~mm}$ vs. $21.83 \pm 5.14 \mathrm{~mm}, \mathrm{p} 0.82)$, the mean left atrial volume was also bigger $(61.07 \pm 25.19 \mathrm{ml}$ vs. $59.65 \pm 22.28 \mathrm{ml}, \mathrm{p} 0.85)$ and the mean left ventricular ejection fraction was slightly better than at the patients without progression $(59.23 \pm 4.13 \%$ vs. $58.52 \pm 3.71 \%$, p 0.53).

Regarding the grade of LVDD of the 21 patients, there was no statistically significant association between the E/A ratio and the progression to a sustained form of $\mathrm{AF}$. The number of patients with 3rd grade LVDD that progressed

Table I. Baseline echocardiography characteristics of the study group

\begin{tabular}{lc}
\hline Echocardiographic baseline characteristics & Value \\
\hline Mean LA diameter $(\mathrm{mm})$ & $21.91 \pm 7.13$ \\
Mean LA volume $(\mathrm{ml})$ & $60.17 \pm 23.18$ \\
Mean LA volume/BSA $\left(\mathrm{ml} / \mathrm{m}^{2}\right)$ & $30.8 \pm 11.84$ \\
Mean LV ejection fraction $(\%)$ & $58.78 \pm 3.95$ \\
Diastolic dysfunction 1 & 40 \\
Diastolic dysfunction 2 & 13 \\
Diastolic dysfunction 3 & 4 \\
\hline
\end{tabular}

LA-left atrium; BSA-body surface area; LV-left ventricle 


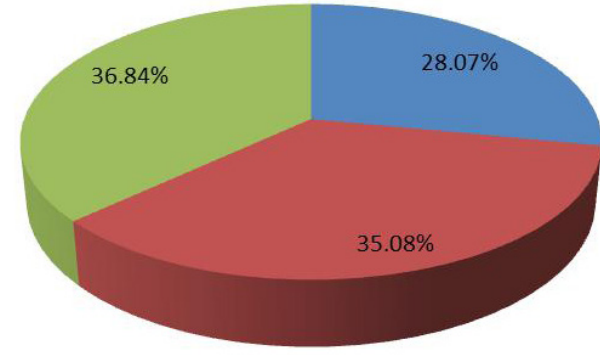

- Paroxysmal $\mathrm{AF}$

- Persistent AF

armenent AF

Fig. 2. AF types at 6 months follow-up

to permanent AF was the same with the patients that did not progress (figure 3).

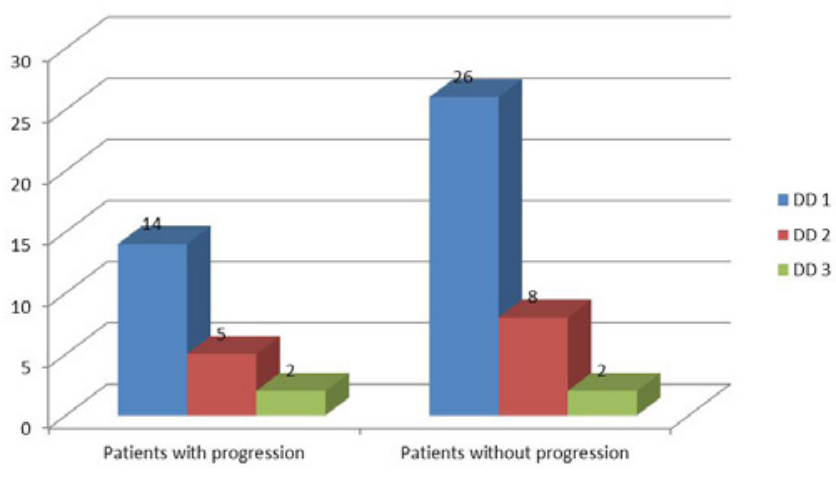

Fig. 3. Progression to permanent AF according to the grade of diastolic dysfunction (DD)

\section{Discussions}

One of the first changes that characterize left ventricular diastolic dysfunction is impaired relaxation, meaning that less ventricular filling occurs in early diastole, and due to atrial contraction, the ventricle will fill more in late diastole. This leads to an increase in the amount of work done by the atrium. Furthermore, due to continuous increase of pressure in the left atrium, a state of restrictive filling occurs and the left ventricle filling will be done rapidly, with minimal contribution from the atria. By this point, the ventricle fills only in the beginning of the diastole, for a brief period, and often symptoms of heart failure are present. The high LV filling pressures might have a role in the future atrial remodeling, which in turn may lead to atrial fibrillation development or progression $[5,6,7]$.

The link between diastolic dysfunction and an increase in left atrial size is proved, and also the increased left atrial size was demonstrated in more than one study to be responsible for the onset, or progression to sustained forms, of atrial fibrillation $[8,9]$. However the number of studies on patients with left ventricular diastolic dysfunction and atrial fibrillation is limited [10]. In a recent study, Kumar et al [11] found that grade 2 or 3 LVDD was an independent predictor for recurrence of atrial fibrillation after ablation.

In the current study we tried to prove the potential link between LVDD, which leads to atrial size increase and progression from paroxysmal or persistent to permanent AF. The studied group was limited, only a small number of patients met the inclusion criteria. Theoretically the tie between left ventricular diastolic dysfunction and atrial fibrillation exists, but remains to be demonstrated.

\section{Conclusion}

Our results showed that female patients with age over 65 had more often atrial fibrillation, but more men progressed to a sustained form of AF. The grade of left ventricular diastolic dysfunction did not associate with the progression to permanent atrial fibrillation, neither the left atrial size or volume, or the left ventricular ejection fraction.

\section{Conflicts of interest}

None declared

\section{References}

1. Colilla S, Crow A, Petkun W et al. Estimates of current and future incidence and prevalence of atrial fibrillation in the U.S. adult population. Am J Cardiol, 2013;112:1142-1147.

2. January CT, Wann S, Alpert JS et al. 2014 AHAVACC/HRS Guideline for the Management of Patients With Atrial Fibrillation. J Am Coll Cardiol, 2014; 10.1016e:11-e14

3. Camm AJ, Kirchhof P, Lip GY et al. Guidelines for the management of atrial fibrillation: the Task Force for the Management of Atrial Fibrillation of the European Society of Cardiology (ESC). Eur Heart J, 2010;31:23692429.

4. Wang TJ, Larson MG, Levy D et al. Temporal relations of atrial fibrillation and congestive heart failure and their joint influence on mortality: the Framingham Heart Study. Circulation, 2003;107:2920-2925.

5. Rosenberg MA, Manning WJ. Diastolic dysfunction and risk of atrial fibrillation. A mechanistic appraisal. Circulation, 2012;126:2353-2362.

6. Otto CM. Ventricular diastolic filling and function. Textbook of Clinical Echocardiography. Saunders Elsevier, 2009:157-181.

7. Meta-analysis research group in echocardiography (MeRGE) heart failure collaborators. Independence of restrictive filling pattern and LV ejection fraction with mortality in heart failure: an individual patient metaanalysis. Eur J Heart Fail, 2008;10:786-792.

8. Schotten $U$, Neuberger HR, Allessie MA. The role of atrial dilatation in the domestication of atrial fibrillation. Prog Biophys Mol Biol, 2003;82:151162

9. Tsang TS, Gersh BJ, Appleton CP et al. Left ventricular diastolic dysfunction as a predictor of the first diagnosed nonvalvular atrial fibrillation in 840 elderly men and women. J Am Coll Cardiol, 2002;40:1636-1644.

10. Kang H, Bae BS, Kim JH et al. The relationship between chronic atrial fibrillation and reduced pulmonary function in cases of preserved left ventricular systolic function. Korean Circ J, 2009;39:372-377.

11. Kumar P, Patel A, Mounsey JP et al. Effect of Left Ventricular Diastolic Dysfunction on Outcomes of Atrial Fibrillation Ablation. Am J Cardiol, 2014;:S0002-9149(14)01120-5. doi: 10.1016 